\title{
Abundancia relativa, estructura poblacional, preferencia de hábitat y patrones de actividad del tapir centroamericano Tapirus bairdii (Perissodactyla: Tapiridae), en la Selva de Los Chimalapas, Oaxaca, México
}

\author{
Iván Lira-Torres, Miguel Briones-Salas ${ }^{1} \&$ Gerardo Sánchez-Rojas ${ }^{2}$ \\ 1. Centro Interdisciplinario de Investigación para el Desarrollo Integral Regional, Unidad Oaxaca (CIIDIR-OAX.), IPN. \\ Hornos 1003, Santa Cruz Xoxocotlán, Oaxaca, Oax. México, C.P.71230; ilira_12@hotmail.com, \\ miguelbrionessalas@hotmail.com \\ 2. Instituto de Ciencias Básicas e Ingeniería, Universidad Autónoma del Estado de Hidalgo, Centro de Investigaciones \\ Biológicas. A.P. 69, Pachuca, Hidalgo, C.P. 42001, México;gerardo.sanchezrojas@gmail.com
}

\author{
Received 02-XII-2013. Corrected 25-IV-2014. Accepted 27-V-2014.
}

\begin{abstract}
Relative abundance, population structure, habitat preferences and activity patterns of Tapirus bairdii (Perissodactyla: Tapiridae), in Chimalapas forest, Oaxaca, Mexico. Baird's tapir (Tapirus bairdii) is endangered primarily because of habitat loss and fragmentation, and overhunting throughout its distribution range. One of the priority land areas for the conservation of this species is the Northern part of its range in the Chimalapas forest, Oaxaca. The aim of this research was to determine the relative abundance, population structure, habitat preferences and activity patterns of Baird's tapir (Tapirus bairdii) in the Chimalapas forest, Oaxaca, Mexico, through the non-invasive technique of camera-trap sampling. A total of five sampling sessions were undertaken among 2009-2013, and used a total of 30 camera-traps in each period. The determinant factor of the sampling design was the hunting between two study areas. A total sampling effort of 9000 trap-days allowed to estimate an index of relative abundance (IRA) of 6.77 tapir photographs/1 000 trap-days $(n=61)$. IRA varied significantly between sampling stations (Mann-Whitney, $\mathrm{p}<0.01$ ). The frequency of Baird's tapir photos was higher in the dry season in tropical rain forest without hunting $\left(x^{2}, \mathrm{p}<0.5\right)$. In the rainy season, the tropical rain forest and secondary vegetation habitats showed higher photo frequency than expected from random $\left(x^{2}, \mathrm{p}<0.5\right)$. Considering population structure, a $95.08 \%$ of adult animals was obtained in photographic records $(\mathrm{n}=58)$. Three types of activity pattern were observed, with more nocturnal records $(88.33 \%$; Kruskal-Wallis, $\mathrm{p}<0.05)$. The Chimalapas forest appears to be the second most important terrestrial priority ecoregion, just after the Mayan Forest (Campeche, Chiapas, Quintana Roo), for the conservation of tapir populations, not only for Mexico but also for Central America. Rev. Biol. Trop. 62 (4): 1407-1419. Epub 2014 December 01.
\end{abstract}

Key words: activity patterns, habitat preferences, relative abundance, Tapirus bairdii, Chimalapa.

El crecimiento acelerado de la población humana ha provocado una explotación inadecuada y descontrolada de los recursos naturales, esto a su vez ha propiciado la extinción de varias especies y puesto en riesgo a otras más (Ceballos, Arroyo-Cabrales, \& Medellín, 2002; Ceballos \& Eccardi, 2003, Cardillo et al., 2005). En México, entre los ecosistemas más amenazados se encuentran los bosques tropicales, en especial el bosque tropical perennifolio, subperennifolio, caducifolio, bosque mesófilo de montaña y el manglar (CONABIO 2009). Se estima que el $70 \%$ de estos bosques se han perdido debido a la deforestación y a modificaciones en el hábitat en los últimos 40 años (CONABIO 2009).

Estos ecosistemas, constituyen el hábitat del único representante del orden Perissodactyla en México: el tapir centroamericano (Tapirus bairdii). Este mamífero neotropical, se distribuyó en tiempos históricos de manera casi continua por la vertiente del Golfo de México, 
desde Veracruz hasta Yucatán (Leopold, 1965; Álvarez del Toro, 1991; Villa \& Cervantes, 2003; Ceballos \& Oliva, 2005), y por el Pacifico, desde Guerrero hasta Chiapas (LiraTorres, Naranjo, \& Chargoy, 2005; Lira-Torres, Naranjo, Hilliar, Camacho, \& De Villa, 2006; Nolasco, Lira-Torres, \& Ceballos, 2007). En la actualidad, se encuentra en la lista roja de especies amenazadas de acuerdo a la Unión Internacional para la Conservación de la Naturaleza (IUCN) (Castellanos et al., 2008), y es considerado en México como en peligro de extinción de acuerdo a las leyes mexicanas; NOM-059-ECOL- 2010 (SEMARNAT, 2010). Además de esto, se encuentra enlistado en el Apéndice I de CITES (CITES, 2013), donde el comercio de individuos de esta especie se permiten solamente en circunstancias excepcionales (donaciones entre instituciones zoológicas internacionales). La alteración del hábitat en todo su rango de distribución está afectando y aislando severamente las poblaciones del tapir centroamericano, que generalmente no ocupan hábitats dominados o modificados por el hombre, y que con frecuencia buscan escapar de las amenazas propiciadas por el ser humano (March, 1994; Naranjo, 2009).

Se estima que la disminución poblacional del tapir centroamericano en los últimos 30 años ha sido mayor al 50\% (CBSG, 2005). Actualmente, la población mundial se estima en 5500 individuos adultos, y se infiere que México cuenta con una población de menos de 1500 ejemplares (Castellanos et al., 2008).

Una de las regiones terrestres prioritarias para la conservación del tapir centroamericano en la zona norte de su rango de distribución se encuentra en la Selva de los Chimalapas, Oaxaca, dentro de la eco-región denominada como Selva Zoque, en los Estados de Oaxaca, Veracruz y Chiapas en México (Nolasco, 2009; Lira-Torres et al., 2006). La Selva de los Chimalapas, es una región extensa (590 993ha), poco explorada, y que contiene la segunda mayor área de bosques tropical perennifolio y subperennifolio en buen estado de conservación de México, después de la Selva Maya. A pesar de esta importancia, en la región no se han decretado áreas del Sistema Nacional de Áreas Naturales Protegidas de México, y aunque empiezan a formarse áreas comunitarias de conservación, sigue existiendo una fuerte presión sobre sus recursos naturales (Ortega del Valle, Carranza, \& Martínez, 2012).

El estado de las poblaciones del tapir centroamericano es poco conocido en la mayor parte de su distribución, pues existen pocas estimaciones de abundancia y densidad. Esto puede deberse al difícil acceso a las áreas de distribución actual de la especie y a la dificultad para observarlo en la vida silvestre (Naranjo, 2009). Tradicionalmente se le ha estudiado utilizando métodos indirectos, donde la habilidad del observador, la colocación de los transectos (de ancho fijo o variable), el intemperismo, el sustrato y la cobertura vegetal, son factores que pueden generar sesgos muy importantes en las estimaciones, lo que dificulta las posibles comparaciones de estos trabajos. Existen cinco trabajos publicados en diferentes áreas naturales sobre la abundancia del tapir centroamericano en México; sin embargo, los reportes se hacen en unidades distintas (unos en kilómetros recorridos y otros en $100 \mathrm{~km}$ ), y en algunos, se dividen en huella y excretas, y en otros no son consideradas (Naranjo \& Cruz, 1998; LiraTorres, Naranjo, Güiris, \& Cruz, 2004; Naranjo \& Bodmer, 2002; Reyna-Hurtado \& Tanner, 2007; Pérez \& Matus, 2010).

Una manera de evitar estos sesgos, es que en la actualidad se ha incrementado el uso de tecnología nueva, que abarata y facilita la utilización de cámaras trampa (Long, Mackay, Zielinski, \& Ray, 2008). Aunque este sistema en parte requiere de cierta habilidad, es mucho más fácil de estandarizar para hacer monitoreos en diferentes localidades, regiones o países enteros sobre especies prioritarias para la conservación (p. ej. CENJAGUAR-México) (Chávez, Ceballos, Medellín, \& Zarza, 2007).

Con el fin de contribuir a generar información básica del tapir centroamericano en una de las zonas más norteñas de su rango de distribución, los objetivos de esta investigación fueron: determinar la abundancia relativa en el tiempo y la estructura poblacional, así como 
la preferencia de hábitat y los patrones de actividad diarios del tapir centroamericano en la Selva de los Chimalapas, Oaxaca, México, mediante la técnica de cámaras trampa.

\section{MATERIALES Y MÉTODOS}

Área de estudio: El trabajo se realizó dentro de los terrenos comunales de la Congregación de La Fortaleza, Municipio de Santa María Chimalapa, en la región denominada como Los Chimalapas. La cual se ubica al sureste del estado de Oaxaca en la región del Istmo de Tehuantepec (17 $\left.09^{\prime} 32.2^{\prime \prime} \mathrm{N}-94^{\circ} 13^{\prime} 45.3^{\prime \prime} \mathrm{W}\right)$. Limita al este con la Sierra del Espinazo del Diablo, al sur con la Sierra Atravesada, ambos en Oaxaca, al norte con Los Uxpanapas, Veracruz y al oeste con el río Oaxaca, Oax. (Arriaga et al., 2000).

La Congregación de La Fortaleza fue fundada en 1983 por gente procedente de Tuxtepec, Oaxaca y pobladores del municipio Catemaco, Veracruz. Está rodeada por montañas pedregosas y cañadas en las que predomina el bosque tropical perennifolio y subperennifolio, vegetación secundaria y pastizales introducidos (Gobierno del estado de
Oaxaca, 1990; Rzedowski, 1991; Torres-Colín, 2004). El clima es cálido húmedo con lluvias en verano; Am(f) y (A)C(w2) (García, 1973). La precipitación y temperatura media anual varían de 3000 a $3500 \mathrm{~mm}$ y de 18 a $22^{\circ} \mathrm{C}$, respectivamente (Arriaga et al. 2000). El periodo de secas únicamente abarca cuatro meses (marzo-junio). El periodo de lluvia abarca ocho meses (julio-febrero), ya que se combina las lluvias con la temporada de nortes, siendo esta región una de las más húmedas del país (Wendt, 1989). Los suelos son de tipo eútrico (Alfaro, 2004). El sistema hidrográfico es alimentado por las vertientes septentrionales de los ríos Oaxaca, Uxpanapa, y del escurrimiento de la zona central del río Coatzacoalcos (Ortiz Pérez et al., 2004) (Fig. 1).

En la Fortaleza viven cerca de 120 personas, agrupadas en 30 familias. Todos hablan español y algunos pobladores dominan algún idioma indígena. La mayoría de los jóvenes y adultos de la congregación se dedican a la ganadería extensiva de doble propósito, la cual es una de las actividades más recurrentes de la región. De forma temporal cultivan hortalizas (maíz, fríjol y calabaza), como forma de subsistencia. Adicionalmente, y para complementar

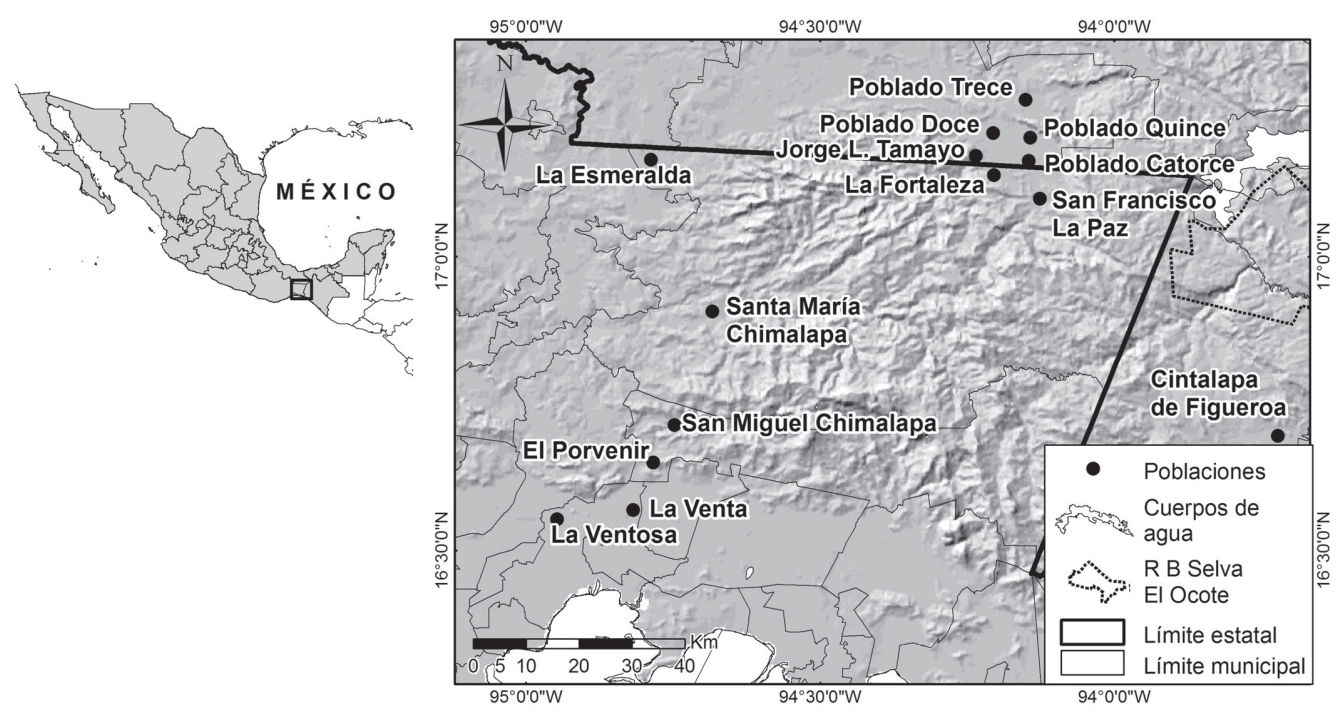

Fig. 1. Selva de los Chimalapas, Oaxaca, México.

Fig. 1. Study site in Chimalapas forest, Oaxaca, Mexico. 
la proteína de origen animal que compone su dieta se practica la cacería de subsistencia. Esta actividad la realizan comúnmente en los fragmentos y/o islas de vegetación secundaria que son dejados por los ganaderos intencionalmente; además, ocasionalmente cazan en el continuo del bosque tropical, especies como: cabro de monte (Mazama temama), saíno (Pecari tajacu), chancho de monte (Tayassu pecari), paca (Cuniculus paca), armadillo (Dasypus novemcintus), coatimundi (Nasua narica), pavón grande (Crax rubra) y la pava crestada (Penelope purpurascens) (Gobierno del estado de Oaxaca,1990; Lira-Torres \& Briones-Salas, 2011).

Diseño de muestreo: Se realizaron cinco periodos de muestreo fotográfico entre 2009 y 2013. El primero se llevó a cabo durante parte de la temporada seca del 2009 (mayojunio), el segundo en la temporada de lluvias del 2010 (julio-agosto), el tercero y cuarto en la temporada de secas (abril-mayo) y lluvias (octubre-noviembre) del 2012, y finalmente, el quinto en la temporada de lluvias (enerofebrero) del 2013. En todos los muestreos se colocaron 30 cámaras trampa durante 60 días de muestreo efectivos. El diseño de muestreo fue el propuesto para evaluar las poblaciones del jaguar (Panthera onca, CENJAGUAR) y sus presas en México; el cual incluye al tapir centroamericano (Chávez et al., 2007). Este diseño consiste en abarcar una superficie de $80 \mathrm{~km}^{2}$ para los sitios prioritarios para la conservación de este depredador neotropical, como lo es nuestro sitio de estudio (Medellín et al., 2006; Lira-Torres \& Ramos-Fernández, 2007; Briones-Salas, Lavariega, \& Lira-Torres, 2012) (Fig. 2).

Las estaciones de muestreo se instalaron en veredas naturales, bordes de montañas, cañadas, arroyos secos y márgenes de río,

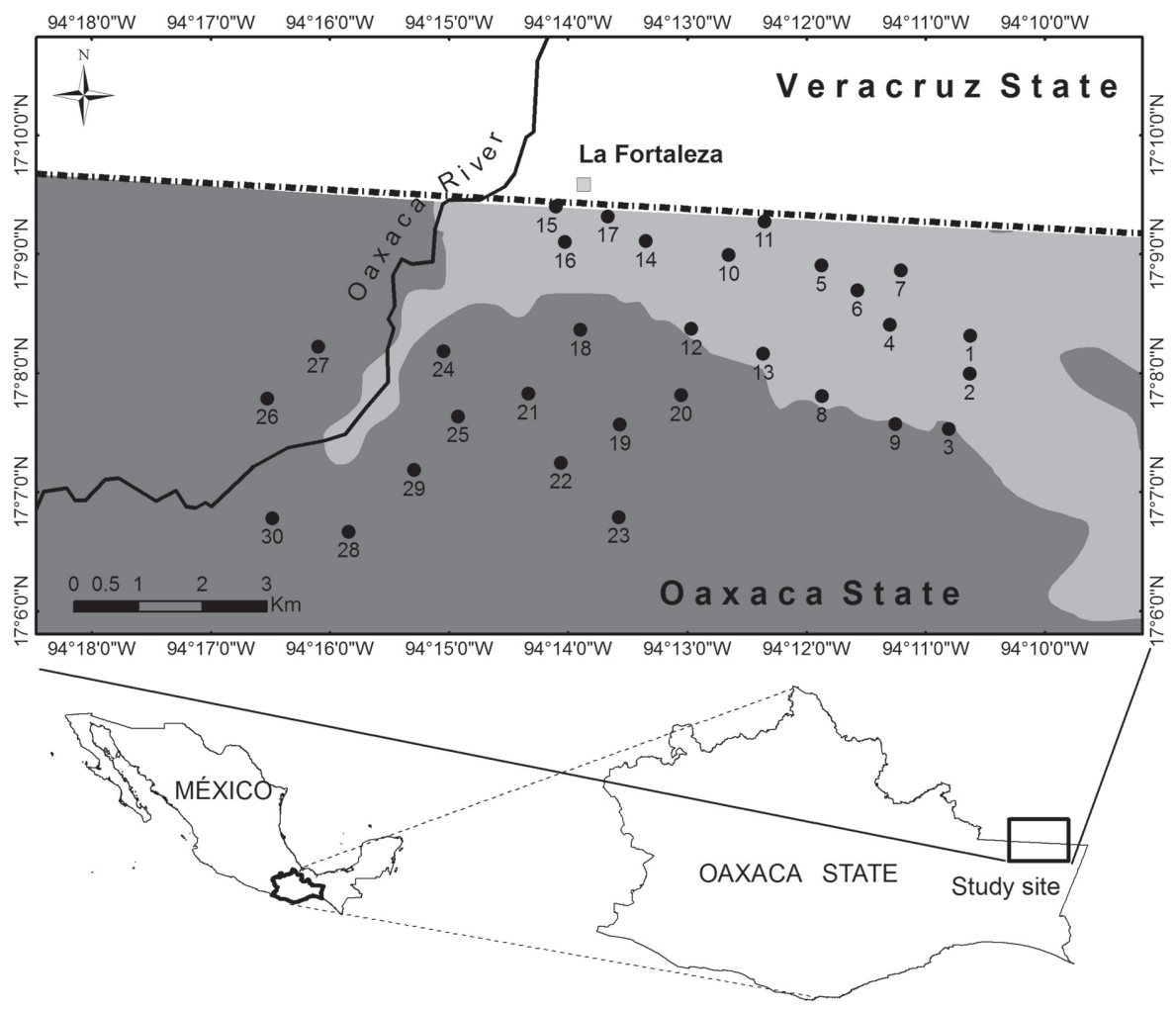

Fig. 2. Distribución de las cámaras trampa en la zona de estudio.

Fig. 2. Camera traps distribution in the study area. 
espaciadas una de otra a una distancia de 1.5 a $3 \mathrm{~km}$ para no dejar áreas mayores sin muestrear, $\mathrm{y}$ asumiendo que esta distancia es lo suficientemente grande para lograr la independencia estadística entre las estaciones de trampeo. En cada estación de muestreo se instaló una cámara trampa modelo Cuddeback Digital ${ }^{\circledR}$, a una altura no mayor de 40 o $50 \mathrm{~cm}$ del nivel del suelo, dependiendo de la topografía e inclinación del área de muestreo. El circuito de estas cámaras fue programado para permanecer activo durante 24 horas. La posición de cada una de ellas fue georeferenciada con un geoposicionador modelo Garmin etrex ${ }^{\circledR}$. Posteriormente, se revisaron una vez al mes y en cada fotografía detectada se imprimió la hora y fecha. El esfuerzo total de muestreo se obtuvo multiplicando el número total de cámaras por el total de días de muestreo (Medellín et al., 2006).

\begin{abstract}
Abundancia relativa: Para obtener el índice de abundancia relativa (IAR) se utilizó una fórmula probada por los trabajos de Maffei, Cuellar, y Noss (2002); Sanderson (2004); Azuara (2005); Jenks et al. (2011) y Zamora (2012):
\end{abstract}

\section{$\mathrm{IAR}=\mathrm{C} / \mathrm{EM}^{*} 1000$ días trampa}

Donde: $\mathrm{C}$ es el número de eventos fotografiados (independientes); EM es el Esfuerzo de Muestreo (medido como número de cámaras * días de monitoreo) Estacional o Total y 1000 días-trampa (Unidad Estándar).

Se consideraron como registros fotográficos independientes los siguientes casos: a) fotografías consecutivas de diferentes individuos, y b) fotografías consecutivas de la misma especie separadas por 24 horas (este criterio fue aplicado cuando no era claro si una serie de fotografías correspondían al mismo individuo, de modo que las fotografías tomadas antes de 24 horas se consideraron como un solo registro) (Maffei et al., 2002; Sanderson, 2004; Azuara, 2005; Jenks et al., 2011; Zamora, 2012). Para observar variaciones entre épocas de muestreo; secas y lluvias, en los índice de abundancia relativa (IAR) obtenidos, se realizaron comparaciones por medio de una prueba de Suma de Rangos de Mann-Whitney (Sokal \& Rohlf, 1995).

Estructura poblacional: Se determinaron las clases de edad y sexo mediante las fotografías obtenidas. Las categorías de edad a considerar fueron: 1) Crías (individuos pequeños acompañados de su madre y con puntos o rayas blancas); 2) Juveniles (individuos solitarios con o sin vestigios de manchas blancas o rayas, pero claramente más pequeños que los adultos); y 3) Adultos (individuos grandes sin rayas o manchas, con muy poco pelo en la grupa (Naranjo \& Bodmer, 2002).

Patrón de actividad: Este fue determinado cuando se obtuvieron al menos 11 registros fotográficos de la especie independientes con la hora visible (Maffei et al., 2002; Monroy-Vilchis, Zarco-González, RodriguezSoto, Soria-Díaz, \& Urios, 2011). Los registros obtenidos se ordenaron por intervalos de una hora. Los patrones de actividad se agruparon en tres unidades: a) diurnos (08:00-18:00hr), cuando en las fotografías se observaba luz solar; b) nocturnos cuando no había luz solar, (20:00-06:00hr), y c) crepusculares, cuando se obtuvieron al amanecer (06:00-08:00hr) o al atardecer (18:00-20:00hr). Para observar variación en los patrones de actividad se realizaron comparaciones por medio de una prueba de Kruskal-Wallis (Sokal \& Rohlf, 1995).

Preferencia de hábitat: Para estimar la preferencia de hábitat en los patrones de ocurrencia del foto trampeo, se consideró que la distribución de las estaciones de muestreo fuera de la siguiente manera: se colocaron siete estaciones en remanentes de vegetación secundaria ó acahuales con presencia de cacería, ocho en remanentes de vegetación secundaria sin presencia de cacería. Además, siete estaciones se colocaron en un continuo de bosque tropical perennifolio con cacería y las ocho restantes en un continuo de bosque tropical perennifolio sin cacería. La preferencia de hábitat se estimó 
por medio del número de registros fotográficos independientes por estación de muestreo. Mediante la prueba de Chi cuadrada, se obtuvo la frecuencia observada, esperada y los intervalos de Bonferroni para cada tipo de cobertura vegetal utilizada por la especie, para lo cual se empleó el programa HABUSE 4.0 (Byers, Steinhorst, \& Krausman, 1984).

\section{RESULTADOS}

El esfuerzo total de muestreo en los cinco periodos fue de 9 000días/trampa; 1 800días/ trampa para cada uno de ellos, en los cuales se obtuvieron 3058 fotografías de mamíferos grandes y medianos de la región, así como de aves. De los registros obtenidos, 61 correspondieron a eventos independientes del tapir centroamericano.

En el primer muestreo (temporada seca 2009) se obtuvieron 2363 fotografías, 24 fotografías de tapir centroamericano como registros independientes. En el segundo muestreo (temporada de lluvia 2010), se obtuvieron 86 fotografías, 17 fueron registros de tapir. En el tercer (temporada seca 2012) y cuarto muestreo (temporada lluviosa 2012), se obtuvieron 308 y 185 fotografías respectivamente, de estas, ocho en la temporada seca y 12 en la lluviosa fueron registros independientes de tapir centroamericano. Finalmente, en el quinto muestreo (temporada de lluvia 2013), se obtuvieron 116 fotografías de mamíferos y aves de la región, de las cuales ninguna foto correspondió a tapires.

Abundancia relativa: Los IAR obtenidos para el tapir centroamericano en cada uno de los cinco periodos de muestreo se presentan a continuación. Para el primer muestreo se obtuvo un $\mathrm{IAR}=13.33$ ( $\mathrm{n}=24)$, en el segundo muestreo de IAR=9.44 $(n=17)$, en el tercer muestreo de IAR=4.44 $(n=8)$, en el cuarto muestreo se obtuvo un IAR=6.66 $(n=12)$, y finalmente en el quinto muestreo, no se obtuvieron fotografías para calcular el IAR. El índice de abundancia relativa total (IAR) para los cuatro años fue de $6.77 / 1000$ trampas-noche $(n=61)$. Los índices de abundancia relativa (IAR) obtenidos variaron significativamente entre épocas de muestreo (Mann-Whitney, $\mathrm{U}=3$ 419, $\mathrm{p}<0.01$ ) (Cuadro 1).

Estructura poblacional: Durante el estudio se obtuvo un $95.08 \%$ de registros fotográficos de animales adultos $(\mathrm{n}=58), 3.27 \%$ de animales jóvenes $(\mathrm{n}=2)$ y el $1.63 \%$ con el registro de una cría $(\mathrm{n}=1)$. También se identificó el sexo de 19 de los 61 registros fotográficos. Se identificaron 16 machos $(26.22 \%)$, tres hembras $(4.91 \%)$ y no se pudo identificar el sexo en 41 de los registros (67.21\%).

Patrón de actividad: Los resultados muestran que $T$. bairdii es una especie nocturna, ya que presentó el mayor porcentaje de

CUADRO 1

Índices de abundancia relativa (IAR) de Tapirus bairdii, en la Selva de los Chimalapas, Oaxaca, México

TABLE 1

Index of relative abundance (IAR) of Tapirus bairdii in the Chimalapas forest, Oaxaca, Mexico

\begin{tabular}{lcc}
\multicolumn{1}{c}{ Fotografías de Tapires Tapirus bairdii en la Zona de Estudio } & Núm. total de fotografías de tapires & IAR \\
Época de secas 2009 & 24 & 13.33 \\
Época de lluvias 2010 & 17 & 9.44 \\
Época de secas de 2012 & 8 & 4.44 \\
Época de lluvias de 2012 & 12 & 6.66 \\
Época de lluvias de 2013 & - & - \\
Total & 61 & 6.77 \\
\hline
\end{tabular}

IAR vs Estacionalidad (Mann-Whitney, $U=3419, \mathrm{p}<0.01$ )* 
registros durante este periodo; $88.33 \%(\mathrm{n}=53)$, con actividad entre las 20:00 a 05:00h (Fig. 3). Se obtuvieron diferencias significativas (Kruskal-Wallis, $\mathrm{H}=3679, \mathrm{p}<0.05)$ en los tres periodos de tiempo (nocturno, diurno y crepuscular).

Preferencia de hábitat: De acuerdo a las fotografías obtenidas durante los periodos de muestreo en la temporada de secas (2009 y 2012), el bosque tropical perennifolio sin cacería fue el hábitat más utilizado que lo esperado por azar $\left(x^{2}=8.822, \mathrm{p}<0.5\right)$. La vegetación secundaria con cacería fue utilizada conforme a lo esperado por su disponibilidad. Finalmente, el bosque tropical perennifolio con cacería; no obstante su extensión, fue utilizado menos de lo esperado por azar (Cuadro 2).

Por otro lado, durante los periodos de muestreo en la temporada de lluvia (2010, 2012 y principios del 2013), el bosque tropical perennifolio con cacería y la vegetación secundaria con cacería fueron los hábitat más utilizados que lo esperado al azar $\left(x^{2}=3.947, \mathrm{p}<0.5\right)$. Mientras que el bosque tropical perennifolio sin cacería, fue el hábitat menos utilizado que lo esperado por la especie al azar (Cuadro 2).

\section{DISCUSIÓN}

Nuestros resultados muestran que el uso de las cámaras trampa es una buena alternativa para estudios de investigación biológica con fines de conservación, que complementa los métodos convencionales de monitoreo, sobre todo porque apoya el estudio de especies con bajas densidades y difíciles de observar, como el tapir centroamericano, cubriendo además un amplio rango de ambientes que otros métodos no permiten, y facilitar la obtención de un número más representativo de datos (Silveira, Jácomoa, \& Diniz-Filhoa, 2003; Pinto de Sá Alves \& Andriolo, 2005; Srbek-Araujo \& García, 2005; Lira-Torres \& Briones-Salas, 2012). Además, estudios recientes con el uso sistematizado de cámaras trampa, han demostrado que se puede estimar la densidad de la población, documentar patrones de actividad (Maffei et al., 2002; Pinto de Sá Alvez \& Andriolo, 2005; Monroy-Vilchis et al., 2011), identificar la preferencia del hábitat (Lira-Torres \& Briones-Salas, 2011), así como describir los patrones de comportamiento de algunas especies (Maffei et al., 2002). No obstante, es

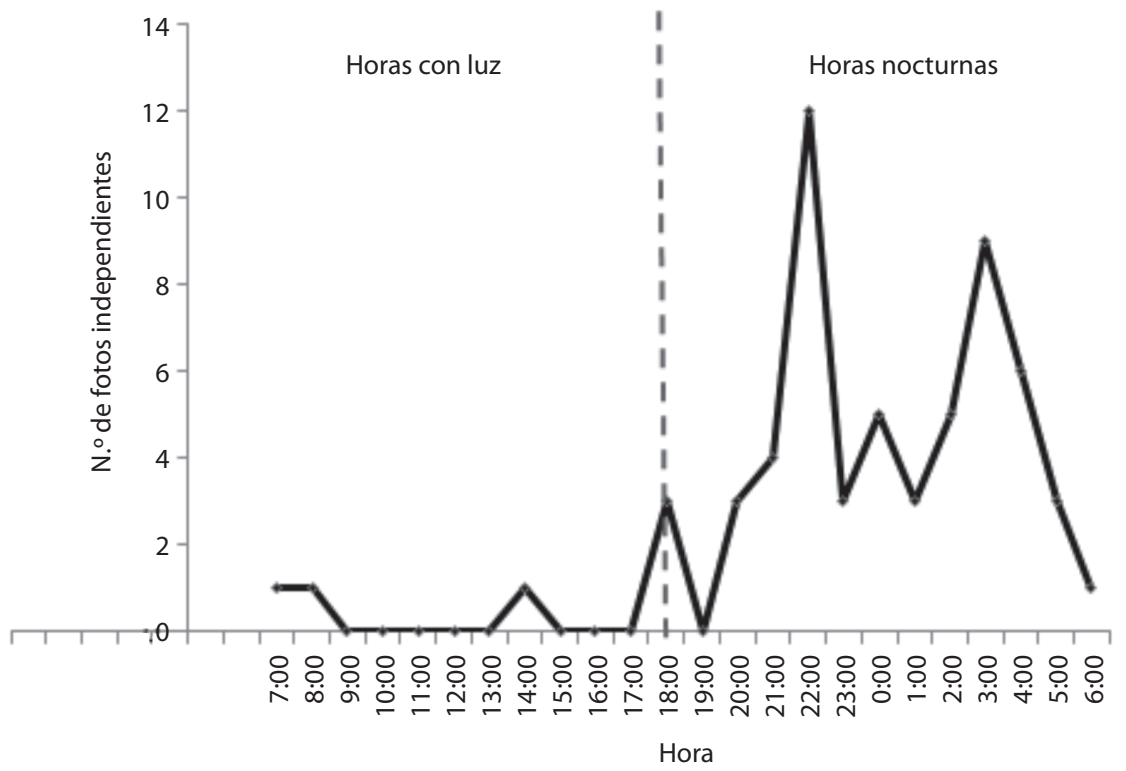

Fig. 3. Patrón de actividad del Tapirus bairdii durante los periodos de muestreo en la Selva de los Chimalapas, Oaxaca, México.

Fig. 3. Activity patterns of Tapirus bairdii during sampling periods in the Chimalapas forest, Oaxaca, Mexico. 
CUADRO 2

Frecuencia esperada ( $\mathrm{Fe}$ ) y observada (Fo) de Tapirus bairdii por tipo de hábitat durante la temporada seca y lluvias en la Selva de los Chimalapas, Oaxaca, México

TABLE 2

Expected frequency (Fe) and observed (Fo) of Tapirus bairdii by type of habitat during the dry and rainy season in the Chimalapas forest, Oaxaca, Mexico

\begin{tabular}{lcccccc}
\multicolumn{1}{c}{ Tipos de Hábitat } & Área (\%) & $\mathrm{Fe}$ & $\mathrm{Pfe}^{1}$ & $\mathrm{Fo}$ & $\mathrm{Pfo}^{2}$ & $\begin{array}{c}\text { Inter. Bonferroni } \\
(\mathrm{p}<0.05)\end{array}$ \\
$\begin{array}{lcccccc}\text { Temporada de Seca } \\
\text { Vegetación Secundaria con Cacería }\end{array}$ & 0.28 & 6.44 & 0.28 & 6 & 0.26 & $0.032-0.490$ \\
Vegetación Secundaria sin Cacería & 0.02 & 0.46 & 0.02 & 0 & 0 & $0.000-0.005$ \\
Bosque Tropical Perennifolio Conservado con Cacería & 0.40 & 9.20 & 0.40 & 4 & $0.17(-)^{3}$ & $0.000-0.371$ \\
Bosque Tropical Perennifolio sin Cacería & 0.30 & 6.90 & 0.30 & 13 & $0.56(+)^{4}$ & $0.307-0.823$ \\
Temporada de Lluvia & & & & & & \\
Vegetación Secundaria con Cacería & 0.25 & 4.75 & 0.25 & 6 & $0.316(+)^{4}$ & $0.060-0.571$ \\
Bosque Tropical Perennifolio con Cacería & 0.50 & 9.50 & 0.50 & 12 & $0.632(+)^{4}$ & $0.367-0.897$ \\
Bosque Tropical Perennifolio Conservada sin Cacería & 0.25 & 4.75 & 0.25 & 1 & $0.53(-)^{3}$ & $0.000-0.175$ \\
\hline
\end{tabular}

Proporción de frecuencias esperadas de fotografías de Tapirus bairdii.

Proporción de frecuencias observadas de fotografías de Tapirus bairdii.

Hábitat significativamente menos utilizado que lo esperado.

Hábitat significativamente igual a lo esperado.

importante considerar que la funcionalidad y efectividad de las cámaras trampa va decayendo conforme la utilización y tiempo de empleo, situación que se documentó en la tendencia hacia la baja de fotografías obtenidas a lo largo de cada periodo y que no necesariamente refleja una disminución en la abundancia de la especies, pues seguía siendo constante el registro de sus rastros (huellas y excretas) (Zamora, 2012).

En el caso de los tapires a nivel mundial, es relativamente reciente y aún escaso el número de publicaciones utilizando esta herramienta para estimar abundancia relativa, y donde además se ha estandarizado el esfuerzo de registros al estimar índices de abundancia relativa (IAR) basado en el número de registros fotográficos/ 1 000 trampas-noches. Lo que permite cierta comparación entre los resultados.

Para el tapir de tierras bajas (Tapirus terrestris) en Sudamérica se ha reportado que la abundancia en el Chaco boliviano es de 11-60 registros/1 000 trampa noches (Noss, Carranza, Barrientos, Maffei, \& Cuellar, 2003); mientras que en bosques tropicales húmedos en Bolivia se obtiene 7 registros/1000 trampa-noche
(Wallace, Ayala, \& Gómez, 2002). En Brasil para otro bosque tropical se estima este índice en 2.71 registros/1 000 trampa-noche (Moraes, Silva, \& Freitas, 2003). Finalmente, Holden, Yanuar, y Martyr (2003) estima 4-19 registros/1 000 trampas-noche para el tapir malayo T. indicus en Sumatra.

En el caso del tapir centroamericano $T$. bairdii se ha reportado 12 registros/ 1000 trampas-noche en los bosques tropicales de Chiquibul Belice (Kelly, 2003), mientras que Pérez-Cortez, Enríquez, Sima-Panti, ReynaHurtado, y Naranjo (2012) y Pérez (2011) estimó un índice 37.57 registros/1 000 trampasnoche en la Reserva de la Biosfera de Calakmul, México.

Basado en la información anterior, los índices de abundancia relativa obtenidos en la Selva de los Chimalapas, Oaxaca, mediante el uso de cámaras trampa (6.77 registros/1000 trampas-noche), es bastante inferior al citado por Pérez-Cortez et al. (2012) y Pérez (2011) para Calakmul. Hay que inferir que posiblemente los datos de estos últimos autores estén sesgados ya que únicamente está monitoreando 
las lagunas (aguadas; nombre local) y sus alrededores, mismas que influyen, en la presencia y recurrencia del tapir en la Reserva de la Biosfera de Calakmul, México, donde los cuerpos de agua son escasos, situación que no ocurre en los Chimalapas, Oaxaca, donde hay abundancia de cuerpos de agua en toda la región, así como la presencia de ríos y lagunas permanentes y suficiente cobertura vegetal.

Por otro lado, los índices obtenidos en los Chimalapas están dentro de los rangos promedios obtenidos a nivel internacional para otras especies de tapires. Este dato puede ser una evidencia del estado de conservación de la población de tapires en los Chimalapas. Consideramos que con el uso de cámaras trampa en estudios biológicos y ecológicos, el índice de abundancia relativa (IAR) puede ser una unidad unificadora que pueda permitir con mayor facilidad la comparación de la abundancia relativa para esta especie en el futuro (Zamora, 2012).

Con respecto a la estructura poblacional, la proporción de sexos en poblaciones de tapir centroamericano en general favorece más a las hembras que a los machos, aunque no se diferencia significativamente del 1:1 (Naranjo, 2009). Sin embargo, en la Selva de los Chimalapas, 16 de los tapires fotografiados fueron machos, tres hembras y 41 animales sin identificar. Por lo que una de las recomendaciones para tener un mejor resultado o un cambio en las tendencias registradas sería la colocación de cámaras pareadas en lugar de simples, ya que con esto se obtendrían una mayor cantidad de registros fotográficos; sin embargo, esto puede incrementar sustancialmente los costos de monitoreo.

Autores como Matola, Cuarón, y RubioTorgler (1997) han mencionado que la estructura de edades en poblaciones del tapir centroamericano en vida libre con baja o nula presión de cacería, generalmente muestra una gran proporción de adultos, mientras que los jóvenes y las crías representan fracciones relativamente pequeñas. Pese a que sí existe una fuerte presión de cacería en la región, esta misma tendencia fue registrada en la presente investigación con el registros de un 95.08\% de animales adultos $(\mathrm{n}=58)$ (Lira-Torres \& Briones-Salas, 2011). Los bajos números de juveniles y crías en poblaciones de tapir centroamericano con baja o nula presión de cacería puede deberse a que: (a) la especie tiene una baja tasa intrínseca de crecimiento poblacional; (b) los individuos son longevos, alcanzando edades de hasta 30 años; y (c) las tasas naturales de mortalidad tienden a ser muy bajas aún en hábitat sin actividad humana. Por lo que no es extraño que solo contemos con el registro fotográfico de una cría durante los años de muestreo efectuados.

Los registros fotográficos obtenidos por las cámaras trampas durante los años de muestreo confirman que los tapires centroamericanos son mamíferos principalmente nocturnos y solitarios, además se tiene evidencias que los individuos de esta especie se agrupan únicamente para el apareamiento o para la crianza. Esto lo pudimos corroborar con algunas fotografías de parejas copulando dentro de pozas de agua, por lo que es de inferir que las fuentes de agua son de suma importancia para la reproducción de la especie. Sin embargo, y con base a los registros obtenidos, ocasionalmente se le pudo observar en el crepúsculo durante la temporada de lluvias. Posiblemente, este comportamiento se deba a la gran cantidad de humedad relativa o lluvias constantes que le permiten a la especie poder moverse sin sufrir los efectos del calor de medio día.

En lo correspondiente a la preferencia de hábitat, los resultados muestran que hay un mayor uso de lo que se espera del bosque tropical perennifolio sin cacería durante la temporada de secas, por lo que se confirma que la disponibilidad de agua, cobertura y alimento son factores indispensables en la selección del hábitat por la especie (Lira-Torres, Naranjo, Güiris, \& Cruz, 2004). Por otro lado, la vegetación secundaria le siguió en cuanto a utilización en la temporada de secas. Estos fragmentos y/o remanentes de bosques tropicales perennifolios de diferentes tamaños son dejados intencionalmente por los ganaderos en la matriz de pastizales de la región y cumplen 
con tres funciones: a) cuidar las fuentes de agua permanentes de la región; lagunas, que abastecen del vital líquido al ganado doméstico y a las comunidades durante las temporadas de secas, b) ofrecer sombra natural y áreas de descanso al ganado y c) como zona de caza, debido a que en su mayoría estos fragmentos están asociados tanto a árboles frutales nativos (e.g. Ficus sp, Pouteria sapota, entre otros), como introducidos (e.g. Citrus sinensis), por lo que es muy recurrente que los tapires los visiten durante la temporada de secas en busca de alimento, agua y cobertura.

Así mismo, las preferencias registradas durante las temporadas de lluvia por el tapir, posiblemente se deban a que durante estos meses hay una mayor presencia de áreas inundadas. Esto ocurre tanto en los remanentes de vegetación secundaria como en los bosques y fragmentos, debido al desbordamiento de las lagunas, pequeños arroyos y ríos de la región, que limitan el acceso del ganado mismo, así como a los pobladores a estas áreas. Estas áreas inundadas favorecen el estado de sucesión primaria en el hábitat, existiendo una gran cantidad de rebrotes y plántulas de árboles, arbustos y hierbas, los cuales son sumamente atractivos y buscados por el tapir durante esta temporada para su alimentación, ya que contienen una gran cantidad de nutrientes concentrados (LiraTorres et al., 2004).

En varios trabajos se ha descrito que el tapir centroamericano no ocupa hábitats dominados o modificados por el hombre, y que con frecuencia busca escapar de las amenazas derivadas del ser humano (March, 1994; Matola et al., 1997; Lira-Torres et al., 2004 y Naranjo, 2009). Sin embargo, nuestros resultados mostraron que en ambas temporadas de secas y lluvias, estas observaciones difieren, ya que en nuestra zona de estudio, el tapir se encontró en áreas con cierto nivel de perturbación (pastizal o vegetación secundaria) debido a la escasa actividad humana y a que no hay una fuerte presión de cacería sobre la especie. Este patrón ya había sido reportado con anterioridad por Reyna-Hurtado \& Tanner (2007) en las regiones aledañas al área natural protegida de
Calakmul, México; estos autores evidencian que las áreas con cacería y con remanentes de vegetación secundaria, son más importantes para las poblaciones de tapir que la misma área natural protegida (ANP), y que se debe prestar atención a la protección de dichas áreas.

Finalmente, las evidencias nos indican que es frecuente fotografíar a esta especie en la Selva de los Chimalapas, Oaxaca. Si bien esperamos que en el futuro se usen índices estandarizados en otras localidades de México, y en todo el rango de distribución, para comparar abundancias, creemos que es importante continuar el esfuerzo de monitoreo de esta especie, con el fin de recabar datos más robustos que estimen parámetros más precisos (como la densidad), que nos permitan probar que esta zona pudiera ser considerada como la segunda región terrestres prioritaria en importancia; después de la Selva Maya (Campeche, Chiapas, Quintana Roo), en la conservación de las poblaciones de tapir centroamericano, no sólo de México, sino de Centroamérica (sensus Nolasco, 2009).

\section{AGRADECIMIENTOS}

Este trabajo no hubiera sido posible sin el apoyo constante de la Congregación de La Fortaleza, Municipio de Santa María Chimalapa, Oaxaca, así como de los Pobladores del Municipio de Uxpanapa, Veracruz. Agradecemos al Programa Bosques Mexicanos: Selva Zoque, del Fondo Mundial para la Naturaleza (WWF), a la Fundación Carlos Slim y a la SIP del IPN (proyecto SIP: 20121142), por el financiamiento otorgado para la realización del presente estudio. Agradecemos de manera muy especial el apoyo brindado por M. C. Lavariega en la elaboración de los mapas. MB-S agradece a la Comisión de Operación y Fomento a las actividades Académicas (COFFA) y al programa de Estímulos al Desempeño a la Investigación (EDI), del Instituto Politécnico Nacional por el apoyo recibido, así como al Sistema Nacional de Investigadores (SNI) por su reconocimiento y apoyo. 


\section{RESUMEN}

El tapir centroamericano (Tapirus bairdii) está en peligro de extinción debido principalmente a la pérdida y fragmentación de su hábitat, y a la cacería sin control en toda su área de distribución. Una de las regiones terrestres prioritarias para la conservación de esta especie en la zona norte de su distribución se encuentra en la Selva de los Chimalapas, Oaxaca. Por lo que el objetivo de la presente investigación fue determinar la abundancia relativa, estructura poblacional, preferencia de hábitat y patrones de actividad del tapir centroamericano (Tapirus bairdii) en la Selva de los Chimalapas, Oaxaca, México, mediante el uso de cámaras trampa. Se realizaron cinco periodos de muestreo fotográfico entre 2009 y 2013 con un total de 30 cámaras trampa en cada periodo. El factor de diseño fue la intensidad de caza entre dos sitios. Con un esfuerzo total de muestreo de 9000 días/trampa se estimó un índice de abundancia relativa (IAR) de 6.77/1 000 trampas-noche $(n=61)$, variando significativamente entre estaciones de muestreo (Mann-Whitney, $\mathrm{p}<0.01$ ). Durante la temporada de secas, los tapires fueron más abundantes y utilizaron con mayor intensidad el bosque tropical perennifolio sin cacería $\left(\mathrm{x}^{2}, \mathrm{p}<0.5\right)$. Mientras que en la temporada de lluvia, el bosque tropical perennifolio con cacería y la vegetación secundaria con cacería fueron los hábitat significativamente más utilizados que lo esperado $\left(\mathrm{x}^{2}, \mathrm{p}<0.5\right)$. Con respecto a la estructura poblacional se obtuvo un $95.08 \%$ de registros fotográficos de animales adultos $(n=58)$. Se registraron tres tipos de patrones de actividad para la especie, siendo el patrón nocturno el que presentó mayor porcentaje de registros; $88.33 \%$ (Kruskal-Wallis, $\mathrm{p}<0.05$ ). Finalmente, con base al número de registros fotográficos y a los resultados obtenidos es posible considerar a la Selva de los Chimalapas como la segunda eco-región terrestre prioritaria en importancia; después de la Selva Maya (Campeche, Chiapas, Quintana Roo), en la conservación de las poblaciones de tapir centroamericano, no sólo de México, sino de Centroamérica.

Palabras clave: abundancia relativa, patrones de actividad, preferencia de hábitat, México, Tapirus bairdii, Selva de Los Chimalapas.

\section{REFERENCIAS}

Alfaro, S. (2004). Suelos. In A. J. García-Mendoza, M. J. Ordóñez, \& M. Briones-Salas (Eds.), Biodiversidad de Oaxaca (pp. 55-65). México: Instituto de Biología, UNAM, Fondo Oaxaqueño para la Conservación de la Naturaleza-World Wildlife Fund.

Álvarez del Toro, M. (1991). Los mamiferos de Chiapas. México: Gobierno del Estado de Chiapas.

Arriaga, L., Espinoza, J. M., Aguilar, C., Martínez, E., Gómez, L., \& Loa, E. (2000). Regiones terrestres prioritarias de México. México: Comisión Nacional para el Conocimiento y Uso de la Biodiversidad.

Azuara, S. D. (2005). Estimación de abundancia de mamiferos terrestres en un área de la Selva Lacandona, Chiapas. (Tesis de Licenciatura). Facultad de Ciencias, UNAM, México. D.F.

Briones-Salas, M., Lavariega, M., \& Lira-Torres, I. (2012). Distribución actual y potencial del Jaguar (Panthera onca) en Oaxaca, México. Revista Mexicana de Biodiversidad, 83, 246-257.

Byers, C. R., Steinhorst, R. K., \& Krausman, P. R. (1984). Clarification of a technique for analysis of utilizationavailability data. Journal Wildlife Management, 48, 1050-1053.

Cardillo, M., Mace, G. M., Jones, K. E., Bielby, J., Bininda Emonds, O. R. P., Sechrest, E., Orme, C. D. L., \& Purvis. A. (2005). Multiple Causes of High Extinction Risk in Large Mammal Species. Science, 309, 1239-1241.

Castellanos, A., Foerester, C., Lizcano, D. J., Naranjo, E., Cruz-Aldan, E., Lira-Torres, I., Samudio, R., Matola, S., Schipper, J., \& Gonzalez-Maya. J. (2008). Tapirus bairdii. En IUCN 2012 (Ed.), IUCN Red List of Threatened Species. Version 2012.2. Recuperado de http://www.iucnredlist.org.

CBSG (IUCN/SSC). (2005). Taller de Conservación de la Danta Centroamericana (Tapirus bairdii) - Evaluación de la Viabilidad de la Población y del Hábitat: Libro Breve. Apple Valley. MN, USA: CBSG.

Ceballos, G., Arroyo-Cabrales, J., \& Medellín, R. A. (2002). Mamíferos de México. In G. Ceballos \& J. A. Simonetti (Eds.), Diversidad y Conservación de los Mamiferos Neotropicales (pp. 377-413). México, D.F.: CONABIO-UNAM.

Ceballos, G. \& Eccardi, F. (2003). Animales de México en Peligro de Extinción. México: Fundación Ingeniero Alejo Peralta y Díaz Ceballos, IBP.

Ceballos, G. \& Oliva, G. (2005). Los Mamíferos de México. México: Fondo de Cultura Económica, CONABIO.

Chávez, C., Ceballos, G., Medellín, R., \& Zarza, H. (2007). Primer Censo Nacional del Jaguar. In G. Ceballos, C. Chávez, R. List, \& H. Zarza (Eds.), Conservación y Manejo del Jaguar en México: Estudios de Caso y Perspectivas (pp. 113-141). Distrito Federal, México: CONABIO-Alianza WWF/Telcel-Universidad Nacional Autónoma de México.

CITES. (2013). Convención sobre el Comercio Internacional de Especies Amenazadas de Fauna y Flora Silvestres (Consultado en Abril del 2013). Recuperado de http://www.cites.org/esp

CONABIO. (2009). El Capital Natural de México. Vol. I. Conocimiento actual de la biodiversidad. México, D.F. : Comisión Nacional para el Conocimiento y Uso de la Biodiversidad. 
García, E. (1973). Modificaciones al sistema de clasificación climática de Köppen. México: Instituto de Geografía, U.N.A.M.

Gobierno del Estado de Oaxaca. (1990). Tequio por Chimalapas. México: Comité Estatal de Planeación para el Desarrollo de Oaxaca, Subcomité Especial del COPLADE para la Microrregión de los Chimalapas, Vocalía Ejecutiva de los Chimalapas.

Holden, J., Yanuar, A., \& Martyr, D. J. (2003). The Asian tapir in Kerinci Sablant National Park, Sumatra: evidence collected through photo-trapping. Oryx, $37,34-40$.

Jenks, K. E., Chanteap, P., Damrongchainarong, K., Cutter, P., Cutter, P., Redford, T., Lynam, A. J., Howard, J., \& Leimgruber, P. (2011). Using relative abundance indices from camera-trapping to test wildlife conservation hypotheses - an example from Khao Yai National Park, Thailand. Tropical Conservation Science, 4, 113-131.

Kelly, M. J. (2003). Jaguar monitoring in the Chiquibul forest, Belize. Caribbean Geography, 1, 19-32.

Leopold, A. S. (1965). Fauna silvestre de México: Aves y mamiferos de caza. México: Inst. Mex. Rec. Nat. Ren.

Lira-Torres, I., Naranjo, P. E., Güiris, A. D., \& Cruz, E. (2004). Ecología del Tapirus bairdii (Perissodactyla: Tapiridae) en la Reserva de La Biosfera "El Triunfo" (Polígono I), Chiapas, México. Acta Zoológica Mexicana (n.s.), 20, 1-21.

Lira-Torres, I., Naranjo P. E., \& Chargoy, M. A. (2005). Ampliación del Área de Distribución de Tapirus bairdii, Gill 1865 (Perissodactyla: Tapiridae) en Oaxaca, México. Acta Zoológica Mexicana (n.s.), 21, 107-110.

Lira-Torres, I., Naranjo J. E., Hilliar, D., Camacho, E. M., \& De Villa, A. (2006). Status and Conservation of Baird's Tapir in Oaxaca, México. Tapir Conservation. Newsletter of the IUCN/SSC Tapir Specialist Group, 15(1), 21-28.

Lira-Torres, I. \& Ramos-Fernández, G. (2007). El Estado del Jaguar en los Chimalapas, Oaxaca. In G. Ceballos, C. Chávez, R. List, \& H. Zarza (Eds.), Conservación y Manejo del Jaguar en México: Estudios de Caso y Perspectivas (pp. 71-80). México D.F.: Conabio-Alianza WWF/Telcel-Universidad Nacional Autónoma de México.

Lira-Torres, I. \& Briones-Salas, M. (2011). Impacto de la ganadería extensiva y cacería de subsistencia sobre la abundancia relativa de mamíferos en la Selva Zoque, Oaxaca, México. Therya, 2(3), 217-244.

Lira-Torres, I. \& Briones-Salas, M. (2012). Abundancia relativa y patrones de actividad de los mamíferos de los Chimalapas, Oaxaca, México. Acta Zoológica Mexicana (n.s.), 28, 566-585.
Long, R. A., Mackay, P., Zielinski, W. J., \& Ray, J. C. (2008). Noninvasive survey methods for carnivores. USA: Island Press.

Maffei, L., Cuellar, E., \& Noss, J. (2002). Uso de trampas cámara para la evaluación de mamíferos en el ecotono Chaco-Chiquitanía. Revista Boliviana de Ecología y Conservación Ambiental, 11, 55-65.

March, I. J. (1994). Situación actual del tapir en México [Monografía]. Centro de Investigaciones Ecológicas del Sureste, 1, 1-37.

Matola, S., Cuarón, A. D., \& Rubio-Torgler, H. (1997). Status and action plan of Bird's tapir (Tapirus bairdii). In D. M. Brooks, R. E. Bodmer, \& S. Matola (Eds.), Tapirs: status survey and conservation action plan (pp. 29-45). IUCN, Gland, Switzerland and Cambridge, U.K.: IUCN/SSC Tapir Specialist Group.

Medellín, R., Azuara, D., Maffei, L., Zarza, H., Bárcenas, H., Cruz, E., Legaria, R., Lira, I., Ramos Fernández, G., \& Ávila, S. (2006). Censos y Monitoreo. In C. Chávez \& G. Ceballos (Eds.), El Jaguar Mexicano en el Siglo XXI: Situación Actual y Manejo (pp. 25-35). México. D.F.: CONABIO-ALIANZA WWF TELCEL-Universidad Nacional Autónoma de México.

Monroy-Vilchis, O., Zarco-González, M., Rodriguez-Soto, C., Soria-Díaz, L., \& Urios, V. (2011). Fototrampeo de mamíferos en la Sierra Nanchititla, México. Revista de Biología Tropical, 59, 373-383.

Moraes, E. A., Silva, J., \& Freitas, R. L. A. (2003). The lowland tapir in the Caraca Reserve, Minas Gerais State, Brazil: Preliminary Results. Tapir Conservation. Newsletter of the IUCN/SSC Tapir Specialist Group, 12, 20-22.

Naranjo, E. J. \& Cruz, E. (1998). Ecología del Tapir (Tapirus bairdii) en la Reserva de la Biosfera La Sepultura, Chiapas, México. Acta Zoológica Mexicana (n.s.), 73, 111-125.

Naranjo, E. J. \& Bodmer, R. (2002). Population Ecology and Conservation of Baird's Tapir (Tapirus bairdii) in the Lacandon Forest, Mexico. Tapir Conservation. Newsletter of the IUCN/SSC Tapir Specialist Group, 11(2), 25-33.

Naranjo, E. J. (2009). Ecology and Conservation of Baird's tapir in Mexico. Journal Tropical Conservation Science, 2, 140-158.

Nolasco, A., Lira-Torres, I., \& Ceballos, G. (2007). Ampliación del Área de Distribución Histórica del Tapir (Tapirus bairdii) en el Pacífico Mexicano. Revista Mexicana de Mastozoología, 11, 91-94.

Nolasco, A. L. (2009). Distribución Actual y Estado de Conservación del Tapir Centroamericano Tapirus bairdii Gill, 1865 (Perissodactyla: Tapiridae) en México. (Tesis de Licenciatura). Facultad de Ciencias, UNAM, México. 
Noss, A. J., Carranza, R. L., Barrientos, J., Maffei, L., \& Cuellar, E. (2003). A camera trapping and radio telemetry study of lowland tapir (Tapirus terrestris) in Bolivia dry forests. Tapir Conservation. Newsletter of the IUCN/SSC Tapir Specialist Group, 12(1), 24-32.

Ortega del Valle, D., Carranza, L. T., \& Martínez, J. (2012). Una Mirada desde el Corazón de la Jícara de Oro (Experiencias de Conservación en la Selva Zoque de los Chimalapas). México, D.F.: WWF - México / USAID.

Ortiz-Pérez, M. A., Hernández-Santana, J. R., \& Figueroa, J. M. (2004). Reconocimiento Fisiográfico y Geomorfológico. In A. J. García-Mendoza, M. J. Ordóñez, \& M. Briones-Salas (Eds.), Biodiversidad de Oaxaca (pp. 43-54). México: Instituto de Biología, UNAM, Fondo Oaxaqueño para la Conservación de la Naturaleza-World Wildlife Fund.

Pérez, C. \& Matus, E. (2010). El Tapir Tapirus bairdii en la región sureste del Área de Protección de Flora y Fauna Bala'an Ka'ax, Quintana Roo, México. Therya, 1, 137-144.

Pérez, C. (2011). Distribución y abundancia del tapir (Tapirus bairdii) en la Reserva de la Biosfera Calakmul, México (Tesis de Maestría). El Colegio de La Frontera Sur, México.

Pérez-Cortez, S., Enríquez, P. L., Sima-Panti, D., ReynaHurtado, R., \& Naranjo, E. J. (2012). Influencia de la disponibilidad de agua en la presencia y abundancia de Tapirus bairdii en la selva de Calakmul, Campeche, México. Revista Mexicana de Biodiversidad, 83, 753-761.

Pinto de Sá Alves, L. C. \& Andriolo, A. (2005). Camera traps used on the mastofaunal survey of Araras Biological Reserve, IEF-RJ. Revista Brasileira Zootecnia, 2, 231-246.

Reyna-Hurtado, R. \& Tanner, G. (2007). Ungulate relative abundance in hunted and non hunted sites in Calakmul Forest (Southern Mexico). Biodiversity and Conservation, 16, 743-756.

Rzedowsky, J. (1991). Vegetación de México. México: Limusa.

Sanderson, J. G. (2004). Protocolo para Monitoreo con Cámaras para Trampeo Fotográfico. Tropical
Ecology Assessment and Monitoring (TEAM) Initiative. Conservación Internacional, USA: The Center for Applied Biodiversity Science (CABS).

SECRETARÍA DE MEDIO AMBIENTE, RECURSOS NATURALES (SEMARNAT). (2010). Norma Oficial Mexicana NOM-059-ECOL-2010. Protección ambiental, especies de flora y fauna silvestres de México, categorías de riesgo y especificaciones para su inclusión, exclusión o cambio, y lista de especies en riesgo. Diario Oficial de la Federación, Jueves 30 de diciembre de 2010, 1, 1-77.

Silveira, L., Jácomoa, A., \& Diniz-Filhoa, J. (2003). Camera trap, line transect census and track surveys: a comparative evaluation. Biological Conservation, 114, 351-355.

Sokal, R. R. \& Rohlf, J. F. (1995). Biometry. Salt Lake City, Utah, USA: W.H. Freeman and Co.

Srbek-Araujo, A. C. \& García, A. (2005). Is camera trapping an efficient method for surveying mammals in Neotropical forests? A case study in south-eastern Brazil. Journal of Tropical Ecology, 21, 121-125.

Torres-Colín, R. (2004). Tipos de Vegetación. In A. J. García-Mendoza, M. J. Ordoñez, \& M. BrionesSalas (Eds.), Biodiversidad de Oaxaca (pp. 105-117). México: Instituto de Biología, UNAM, Fondo Oaxaqueño para la Conservación de la Naturaleza, World Wildlife Fund.

Villa, B. \& Cervantes, F. A. (2003). Los Mamíferos de México. México: Editorial Iberoamérica.

Wallace, R., Ayala, G., \& Gómez, H. (2002). Lowland tapir activity patterns and capture frequencies in lowland moist tropical forest. Tapir Conservation. Newsletter of the IUCN/SSC Tapir Specialist Group, 11(14).

Wendt, T. (1989). Las Selvas de Uxpanapa, VeracruzOaxaca, México: Evidencia de Refugios Florísticos Cenozoicos. Anales del Instituto de Biología. Serie Botánica, 58, 29-54.

Zamora, J. (2012). Manual Básico de Fototrampeo: Aplicaciones al estudio de los vertebrados terrestres. Técnicas en Biología de la Conservación - $N^{o} 4$. Valencia, España: Tundra Ediciones. 
